\title{
Plasticity Compartments in Basal Dendrites of Neocortical Pyramidal Neurons
}

\author{
Urit Gordon, ${ }^{\star}$ Alon Polsky, ${ }^{*}$ and Jackie Schiller \\ Department of Physiology, Technion Medical School, Haifa 31096, Israel
}

Synaptic plasticity rules widely determine how cortical networks develop and store information. Using confocal imaging and dual site focal synaptic stimulation, we show that basal dendrites, which receive the majority of synapses innervating neocortical pyramidal neurons, contain two compartments with respect to plasticity rules. Synapses innervating the proximal basal tree are easily modified when paired with the global activity of the neuron. In contrast, synapses innervating the distal basal tree fail to change in response to global suprathreshold activity or local dendritic spikes. These synapses can undergo long-term potentiation under unusual conditions when local NMDA spikes, which evoke large calcium transients, are paired with a "gating molecule," BDNF. Moreover, these synapses use a new temporal plasticity rule, which is an order of magnitude longer than spike timing dependent plasticity and prefers reversed presynaptic/postsynaptic activation order. The newly described plasticity compartmentalization of basal dendrites expands the networks plasticity rules and may support different learning and developmental functions.

Key words: dendrites; plasticity; LTP; dendritic spike; cortex; NMDA

\section{Introduction}

Synaptic plasticity rules widely determine how cortical networks store information. Presently, the best-studied set of plasticity rules is spike timing-dependent plasticity (STDP), in which plasticity is mediated by the global postsynaptic signal of backpropagating action potentials (BAPs) (Paulsen and Sejnowski, 2000; Sjostrom et al., 2002; Dan and Poo, 2004). Previous studies from pyramidal neurons revealed that active and passive properties vary along apical dendrites giving rise to functional differences between proximal and distal dendritic regions (Schiller et al., 1997; Stuart et al., 1997; Larkum et al., 1999a; Magee, 1999, 2000; Migliore et al., 1999; Johnston et al., 2000; Korngreen and Sakmann, 2000). Plasticity rules may also differ along the dendritic tree. Previous studies reported that STDP rules depend on the site of the synapse along the apical dendritic tree of neocortical pyramidal neurons (Froemke et al., 2005; Letzkus et al., 2006; Sjostrom and Hausser, 2006). In addition, synapses located in distal apical dendrites can use local dendritic spikes as the postsynaptic signal to induce plasticity (Golding et al., 2002).

The dendritic tree of pyramidal neurons is subdivided into the apical and basal arborizations. The majority of synaptic inputs to neocortical pyramidal neurons are received and processed by the thin basal dendrites (Larkman, 1991). Despite the importance of basal dendrites the plasticity rules in these dendrites in general

\footnotetext{
Received Aug. 12, 2006; revised 0ct. 20, 2006; accepted 0ct. 20, 2006.

This work was supported by the National Institutes of Health, The Israel Science Foundation, and the Rappaport Foundation. We thank M. Hausser, M. London, B. W. Mel, Y. Poirazi, and Y. Schiller for their helpful comments on this manuscript.

*U.G. and A.P. contributed equally to this work.

Correspondence should be addressed to Dr. Jackie Schiller, Technion Medical School, Bat-Galim, Haifa 31096, Israel. E-mail: jackie@tx.technion.ac.il.

DOI:10.1523/JNEUROSCI.3502-06.2006

Copyright $\odot 2006$ Society for Neuroscience $\quad$ 0270-6474/06/2612717-10\$15.00/0
}

and their location dependence in particular are mostly unknown. We were especially interested in this question because, in contrast to apical dendrites, distal basal dendrites typically support initiation of NMDA spikes that in turn are associated with large local calcium influx (Schiller et al., 2000). As induction of most forms of long-term synaptic plasticity requires calcium influx via activated NMDA-receptor channels (Artola and Singer, 1993; Bliss and Collingridge, 1993; Franks and Sejnowski, 2002; Sjostrom and Nelson, 2002), we hypothesized that NMDA spikes can serve as a local postsynaptic signal for induction of long-term potentiation (LTP), especially in distal basal dendrites, in which these spikes are more easily initiated. Moreover, the unique properties of the NMDA spike, especially with respect to its long duration and highly localized nature (Schiller et al., 2000), are expected to result in different plasticity rules than those in apical dendrites. In this study, we used combined intracellular recordings, calcium imaging, and focal synaptic stimulation in neocortical layer 5 and layer 2-3 pyramidal neurons (Schiller et al., 2000; Polsky et al., 2004) to investigate the plasticity rules along basal dendrites. We report that these rules differ significantly from those reported previously in apical dendrites. Although similar to apical dendrites, synapses innervating proximal basal dendrites undergo LTP in response to pairing EPSPs with BAPs;inputs innervating distal basal dendrites fail to undergo LTP when paired with either BAPs or local spikes and require both initiation of NMDA spikes and a cofactor molecule in the form of BDNF. Moreover, the temporal plasticity rules in distal basal dendrites markedly differ from those reported previously for apical dendrites and those we found in proximal basal dendrites.

\section{Materials and Methods}

Slice preparation and electrophysiological recording. Neocortical parasagittal brain slices from the somatosensory area, $300-350 \mu \mathrm{m}$ thick, were 
prepared from 17- to 24-d-old female and male Wistar rats. Whole-cell patch-clamp recordings were performed from visually identified layer 5 and layer 2-3 pyramidal neurons using infrared-differential interference contrast optics. The extracellular solution contained the following (in mм): $125 \mathrm{NaCl}, 25 \mathrm{NaHCO}_{3}, 25$ glucose, $3 \mathrm{KCl}, 1.25 \mathrm{NaH}_{2} \mathrm{PO}_{4}, 2 \mathrm{CaCl}_{2}$, $1 \mathrm{MgCl}_{2}, \mathrm{pH} 7.4$ at 35 to $36^{\circ} \mathrm{C}$. The intracellular solution contained the following (in mM): $115 \mathrm{~K}^{+}$-gluconate, $20 \mathrm{KCl}, 2 \mathrm{Mg}$-ATP, $2 \mathrm{Na}_{2}$-ATP, 10 $\mathrm{Na}_{2}$-phosphocreatine, 0.3 GTP, 10 HEPES, 0.2 Oregon Green 488 Bapta-1 (OGB-1) or Bapta-6 (OGB-6), pH 7.2. Bicuculline methiodide $(1 \mu \mathrm{M})$ was added to the extracellular solution. The electrophysiological recordings were performed using Multi-Clamp 700A (Molecular Devices, Foster City, CA), and the data were acquired and analyzed using pClamp 8.2 (Molecular Devices), a homemade software, and Igor (Wavemetrics, Lake Oswego, OR) software. Statistical tests were performed using Excel software (Microsoft, Redmond, WA). Measurements are reported as mean \pm SEM. Statistical significance was tested using paired Student's $t$ test.

Focal synaptic stimulation and calcium fluorescence imaging. Focal synaptic stimulation was performed via a theta-patch pipette located in close proximity to the selected basal dendritic segment guided by the fluorescent image of the dendrite. In these experiments, the neurons were filled with the calcium-sensitive dye (OGB-1 or OGB-6, $200 \mu \mathrm{M}$ ) and the basal dendritic tree was imaged with a confocal system (Fluoview 500; Olympus, Tokyo, Japan) mounted on an upright BX51WI Olympus microscope equipped with a $60 \times(0.9$ numerical aperture; Olympus) water objective. The theta-stimulating electrodes were filled with Alexa Fluor 647. The location of the activated inputs was performed as described previously (Polsky et al., 2004). Briefly, small EPSPs (0.5-1 mV at the soma) were evoked and concomitant calcium imaging was performed to located the active dendritic segment and active dendritic spines. Typically, we observed calcium transients that were restricted to a dendritic segment of $10-20 \mu \mathrm{m}$. When a dendrite was activated by two adjacent stimulating electrodes $(30-40 \mu \mathrm{m}$ apart), we monitored the overlap between the two stimulated segments using calcium imaging measurements, as described previously (Polsky et al., 2004).

The extent of the calcium spread was monitored separately for each stimulating electrode. For the electrode that was used to evoke the NMDA spike, the extent of the calcium spread was determined with stimulation intensities just subthreshold to NMDA-spike initiation (once the spike initiated, there was spatial spread of the calcium transient probably caused by secondary activation of voltage-gated calcium channels activated by the large voltage signal). In all experiments, we made sure that the dendritic segments activated by the two stimulating electrodes (as determined by the calcium imaging) did not overlap significantly.

Full images were obtained with a temporal resolution of $1 \mathrm{~Hz}$, and in the line scan mode with a temporal resolution of $512 \mathrm{~Hz}$. Images were analyzed using Tiempo (Olympus), homemade software, and Igor software. Fluorescence changes were quantified as increase in fluorescence from baseline normalized by the baseline fluorescence $(\Delta F / F$ as a percentage). The background florescence was subtracted from all measurements before calculation of the $\Delta F / F$. Calcium transients are reported as mean \pm SD. For comparison of calcium transients in proximal and distal locations, we used a high concentration (0.5-1 mM) of the calcium sensitive dye OGB- 6 and data acquisition was done with a line scan from active dendritic spines.

Destruction of basal branches with laser exposure. Basal branches were destroyed by 2-3 min laser exposure in the line scan mode with both the 488 and $633 \mathrm{~nm}$ lasers. To avoid damage to the soma, the segment damaged was typically $15-25 \mu \mathrm{m}$ away from the soma. The damage was monitored using fluorescence imaging, with damaged branches appearing inflated, "blebby," and saturated with calcium.

Glutamate uncaging. For the uncaging experiments, caged glutamate [4-methoxy-7-nitroindolinyl (MNI)-glutamate; Tocris, San Diego, CA] was photolyzed with a $361 \mathrm{~nm}$ UV-laser beam (Enterprise 2; Coherent, Palo Alto, CA) using point scan mode. The caged glutamate (5-10 mM) was delivered locally to a branch using pressure ejection (5-10 mbar) from an MNI-glutamate-containing electrode ( $2 \mu \mathrm{m}$ diameter).

Long-term plasticity induction protocols. EPSPs were evoked at $0.1 \mathrm{~Hz}$ before and after the induction protocols. Control EPSPs were acquired for 10-20 min before induction. Stability of the experiments was ensured by monitoring the membrane resting potential and the amplitude of the preinduction control EPSPs. In all experiments included, the resting membrane potential was stable within $2 \mathrm{mV}$ and the preinduction control EPSPs (average of six consecutive EPSPs) showed less than a $20 \%$ change. LTP was induced using three different protocols. For pairing NMDA spikes and EPSPs, we typically repeated the pairing 15-30 times at $0.1 \mathrm{~Hz}$. In some experiments, we used only five repetitions or up to 60 repetitions of the induction protocol. In addition we used a theta burst protocol, in which NMDA spikes and EPSPs (2 stimuli at $100 \mathrm{~Hz}$ ) were paired five times at $5 \mathrm{~Hz}$, and this sequence was repeated five times at 0.1 $\mathrm{Hz}$. In these experiments, the stimulating electrode was positioned mainly at the distal half of the dendrites as spike initiation was easier at these dendritic regions. In some of the experiments (mentioned in the text), BDNF (50 $\mathrm{ng} / \mathrm{ml}$ in artificial CSF containing 0.1\% BSA; Alomone Labs, Jerusalem, Israel) was applied via pressure ejection (10-20 mbar) through a glass pipette (tip diameter of $2 \mu \mathrm{m}$ ) locally to a dendrite of interest. The BDNF was applied throughout the induction period only. For pairing protocols, we used three different induction protocols, the first consisted of $30-60$ repetitions at $0.1 \mathrm{~Hz}$ of pairing a train of BAPs at $50-150 \mathrm{~Hz}$ (evoked by short $5 \mathrm{~ms}$ depolarizing somatic current injections) with a single EPSP. The EPSP was evoked $10 \mathrm{~ms}$ before the BAP burst. The second protocol consisted of 15 repetitions at $0.05 \mathrm{~Hz}$ of pairing a burst of BAPs evoked by a $100 \mathrm{~ms}$ depolarizing current injection at the soma with a burst of four EPSPs at evoked at $50 \mathrm{~Hz}$. The EPSP train preceded the BAP burst by $10 \mathrm{~ms}$ (Markram et al., 1997b; Sjostrom et al., 2001). The third induction protocol (theta burst stimulation) consisted of pairing a burst of three BAPs evoked by a depolarizing current injection paired with five EPSPs evoked at $100 \mathrm{~Hz}$. This short burst was repeated five times at $5 \mathrm{~Hz}$ (theta rhythm), and this sequence was repeated five times at $0.1 \mathrm{~Hz}$ (Pike et al., 1999; Golding et al., 2002; Dan and Poo, 2004).

\section{Results \\ Spike timing-dependent plasticity in proximal and distal basal dendrites}

To investigate the rules governing long-term plasticity changes at different locations along basal dendrites, we used the well characterized induction protocol, which consists of pairing EPSPs with BAPs. To differentially activate inputs innervating the proximal and distal portions of basal dendrites, we used focal synaptic stimulation with bipolar theta electrodes combined with confocal fluorescence imaging (Fig. 1A) (Koester and Sakmann, 1998; Schiller et al., 2000; Polsky et al., 2004). Experiments were performed only when focal stimulation evoked well defined calcium transients restricted to a segment of the proximal or distal areas including the identification of active spines. To estimate what fraction of the EPSP originated from the activated dendrite in our experiments, we measured the EPSP amplitude before and after deliberately destroying the activated branch by prolonged intense laser exposure. In these experiments after laser induced destruction of the activated branch, the EPSP amplitude decreased by $80 \pm 14 \%$ for proximal stimulation and $85.1 \pm 11 \%$ for distal stimulation $(n=6 ; p>0.3)$. Hence, we concluded that the majority of the voltage originated from the stimulated target basal branch.

Pairing a train of BAPs (four action potentials, $50-150 \mathrm{~Hz}$ ) with an EPSP generated at the proximal half of basal branches evoked LTP (Fig. 1). Under these conditions, most neurons examined ( 17 of $22 ; 77 \%$ ) were potentiated and the EPSP amplitude increased to an average of $185 \pm 32 \%$ and $215 \pm 39 \%$ of the preinduction control value 40 and $60 \mathrm{~min}$ after induction $(n=$ 22; 10 layer 2-3 and 12 layer 5 neurons). In contrast, the same pairing protocol applied to synapses located at the distal half of basal branches failed to evoke LTP (Fig. 1). In fact, a tendency for 
A
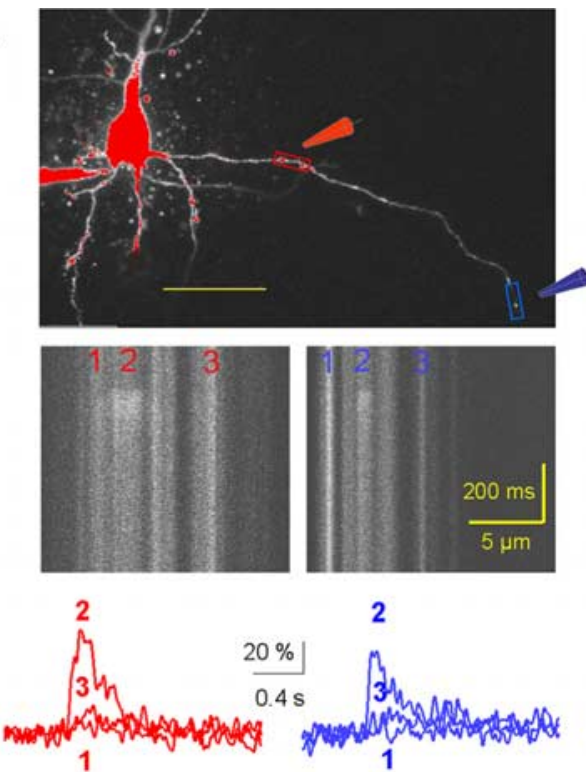

C

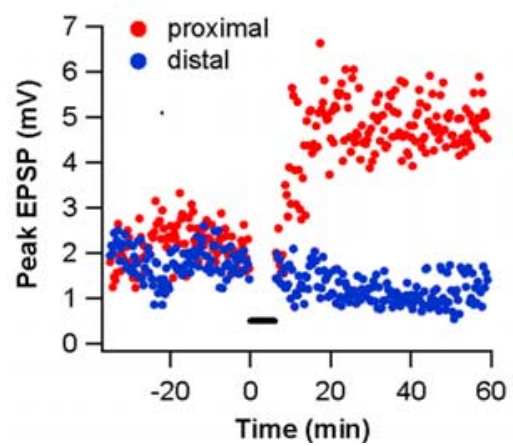

B
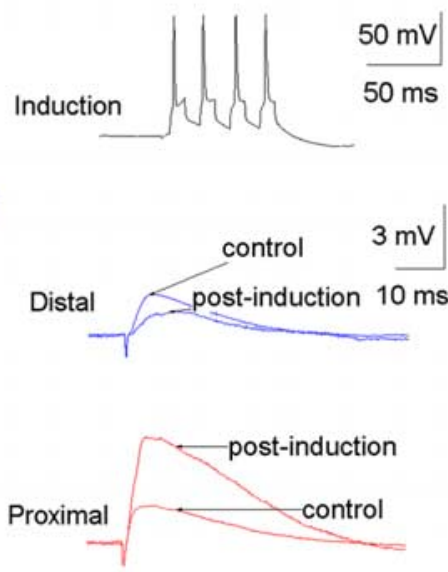

Figure 1. Differential response to pairing induction protocol of proximal versus distal synapses in basal dendrites. $A$, Experimental setup. A layer 5 pyramidal neuron was loaded with OGB-1 $(200 \mu \mathrm{m})$, and two theta-stimulating electrodes were positioned, one proximal (red, $70 \mu \mathrm{m}$ from the soma) and one distal (blue, $200 \mu \mathrm{m}$ from the soma). Calcium transients evoked by small EPSPs (1-2 mV) from proximal (red box) and distal (blue box) regions were collected in the line scan mode. Graphical representation $(\Delta F / F$ as a percentage) is shown for three different regions of the line scan marked by the numbers $1-3$ (red numbers, proximal; blue numbers, distal). The regions marked by 2 show significant calcium transients, whereas in regions 1 and 3 , the calcium transients are markedly diminished. Scale bar, $50 \mu \mathrm{m} . \boldsymbol{B}$, LTP was induced using a pairing protocol consisting of a burst of BAPs (top; 4 action potentials; $50 \mathrm{~Hz}$ ), delivered $10 \mathrm{~ms}$ after a single EPSP. Example EPSP traces (average of 15 traces) before ( $30 \mathrm{~min}$ ) and after ( $50 \mathrm{~min}$ ) induction of the proximal (red) and distal EPSPs (blue) are shown. Note that only the proximally generated EPSPs underwent LTP, whereas the distally generated EPSPs were slightly depressed. C, Peak EPSPs originating from the proximal (red) and distal (blue) were measured simultaneously at the soma before and after induction (black bar, induction period). $\boldsymbol{D}$, Summary of normalized average data from 22 proximal and 14 distal dendritic locations (red, proximal regions; blue, distal regions) shows strong potentiation at proximal synapses and slight depression at distal synapses. Two main reasons caused the slower time course in the averaged potentiation curve compared with the example presented in $\boldsymbol{C}$. First, in some neurons, potentiation occurred gradually. Second, there were differences in the timing of the step-like potentiation between different neurons. Error bars represent SEM.

long-term depression (LTD) was observed. The average EPSP amplitude at these distal locations was $91 \pm 7$ and $85 \pm 9 \%$ of the preinduction control value 40 and $60 \mathrm{~min}$ after induction (Fig. 1) $(n=14 ; 6$ layer $2-3$ and 8 layer 5 neurons; in eight cases, measurements were performed from the proximal and distal regions of the same branch; $p=0.06$ for comparison of the amplitudes obtained 40 and 60 min after induction to the preinduction control value). A similar difference between synapses located proximally and distally was observed with two additional induction protocols: first, pairing a burst of BAPs with a train of four EPSPs following Markram et al. (1997a) $(n=5 ; 94.1 \pm 11.4 \%$ for distal synapses and $178 \pm 33 \%$ for proximal synapses $40 \mathrm{~min}$ after induction) and second, theta-burst stimulation $(n=4 ; 97.3 \pm 14.3 \%$ for distal synapses and $179 \pm 48 \%$ for proximal synapses $40 \mathrm{~min}$ after induction; for more details of stimulation protocols, see Material and Methods). In addition to the focal stimulations, we attempted to evoke LTP by stimulating a broader region of the proximal and distal basal tree. To achieve a broader activation, we positioned an extracellular tungsten electrode 50-100 $\mu \mathrm{m}$ distal to the outer border of the basal tree (for distal activation) and $50 \mu \mathrm{m}$ away from the soma (for proximal activation). Similar to focal stimulation protocols, we observed LTP proximally and not distally $(n=5 ; 92.6 \pm 21.4 \%$ for distal synapses and $198 \pm 38 \%$ for proximal synapses 40 min after induction).

In our experiments, the intracellular solution contained OGB-1 or OGB-6 (200 $\mu \mathrm{M})$ for visualization and localization purposes. These calcium-sensitive dyes introduced extra calcium buffering capacity to the cell. To control for this variable, we replaced the calcium-sensitive dye OGB with Alexa 488, which has no calciumbuffering capacity. No significant differences were observed between experiments with OGB or Alexa dyes in the pipette. When Alexa was present in the pipette 40 min after induction, the average EPSP amplitude was $193 \pm 45 \%$ proximally and $94 \pm 9 \%$ distally $(n=5 ; p>0.3$ compared with OGB experiments).

Most papers in the literature that examined LTP induced by pairing protocols in basal dendrites did not specify the location of activated synapses. However, paired recordings experiments that performed detailed anatomical reconstructions reported that the activated synapses that underwent LTP were mostly located in the proximal portions of basal dendrites (Markram et al., 1997a,b). Thus, our findings regarding proximal basal dendrites are consistent with data in the literature, whereas the literature holds no specific information with regard to synapses located at distal basal dendrites.

\section{Calcium transients in proximal and distal basal dendrites of} layer 5 and layer $2-3$ neurons

Previous studies have shown that induction of LTP is closely linked to the local calcium transients evoked during the induction period (Artola and Singer, 1993; Bear and Kirkwood, 1993; Bliss and Collingridge, 1993; Franks and Sejnowski, 2002; Sjostrom and Nelson, 2002). Thus, we compared the calcium transients evoked by BAPs, EPSPs, and pairing of both in proximal and distal regions of basal dendrites. To minimize dye saturation problems, we performed calcium imaging measurements with high concentration (0.5-1 mM) of the low-affinity dye OGB-6. We found no significant differences in the calcium transients 
evoked by the three protocols between proximal and distal regions of the same basal dendrite (Fig. 2). On average, calcium transients $(\Delta F / F)$ evoked by the LTP pairing protocol were $110 \pm 58 \%$ in eight proximal dendrites and $120 \pm 57 \%$ in 12 distal dendrites $(p>0.5)$. The similarity in the calcium transients in proximal and distal basal dendrites suggested that differences in calcium concentrations were unlikely to account for the inability of inputs in distal basal dendrites to undergo LTP. However, we cannot rule out the possibility that differences in the amplitude or time course of local subcellular calcium concentrations play a role in this phenomenon. It is interesting to note that, in contrast to basal dendrites, in apical dendrites the BAPs induced calcium transients decrease significantly with distance (Spruston et al., 1995; Larkum et al., 1999b).

NMDA spikes are insufficient to induce LTP in basal dendrites of layer 5 and layer 2-3 pyramidal neurons

Dendritic spikes can serve as an alternative postsynaptic signal for the induction of LTP (Golding et al., 2002). We next investigated whether NMDA spikes can serve as the postsynaptic signal for LTP in distal basal dendrites of layers 5 and $2-3$ pyramidal neurons. Because NMDA spikes have only been reported previously for layer 5 neurons, we first characterized these spikes in basal dendrites of layer 2-3 neurons as well. Similar to layer-5 pyramidal neurons (Schiller et al., 2000; Polsky et al., 2004), coactivation of spatially clustered synaptic inputs in basal dendrites of layer 2-3 pyramidal neurons initiated local NMDA spikes (Fig. $3 A, B)$. NMDA spikes in layer $2-3$ pyramidal neurons had an average somatic threshold of $2.94 \pm 0.74 \mathrm{mV}$, average amplitude of $11.06 \pm 5.01 \mathrm{mV}$, and average half-width of $80.4 \pm 38.3 \mathrm{~ms}(n=12)$. Similar to layer 5 neurons, NMDA spikes in layer 2-3 neurons evoked large local calcium transients (Fig. $3 C$ ). On average, the local calcium transients evoked by NMDA spikes were approximately sixfold larger than those evoked by a train of BAPs $(n=9 ; 43 \pm 20$ and $254 \pm 44 \%$ for calcium transients evoked by a train of four action potentials and NMDA spikes in distal basal dendrites, respectively). Similar to layer 5 pyramidal neurons (Schiller et al., 2000), glutamate uncaging onto basal dendrites of layer 2-3 neurons showed that NMDA spikes could be initiated in the presence of voltagegated sodium and calcium channel blockers (TTX $1 \mu \mathrm{m}$, nickel $100 \mu \mathrm{m}$, cadmium 100 $\mu \mathrm{m})$, whereas application of the NMDA receptor blocker APV $(50 \mu \mathrm{m})$ blocked the spike altogether (Fig. 3D).

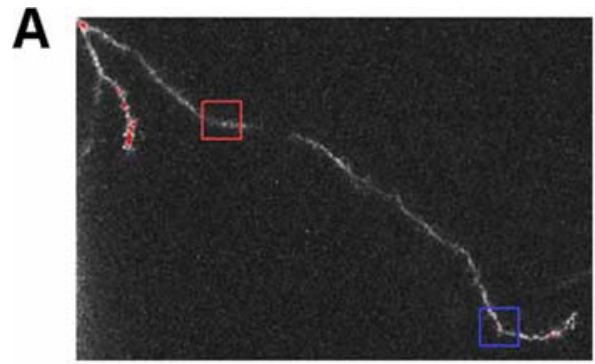

50 microns
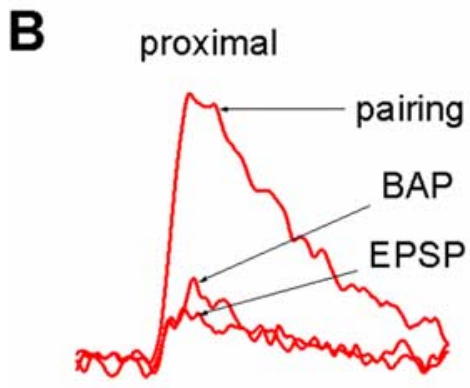
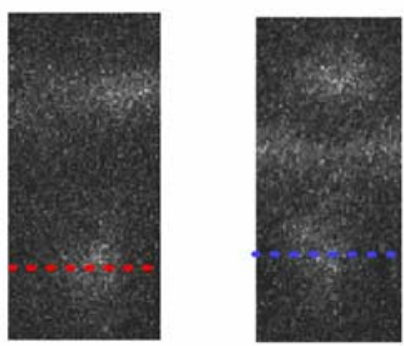

2 microns

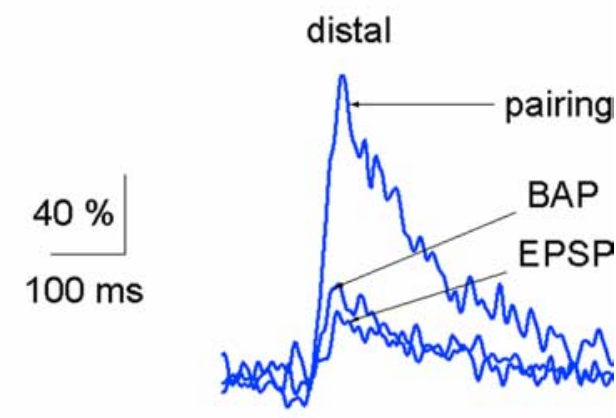

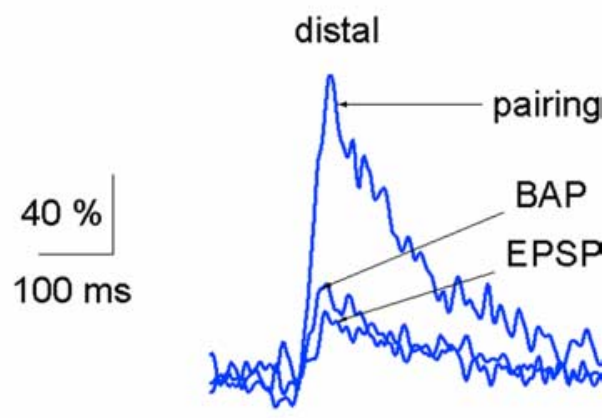

pairing proximal

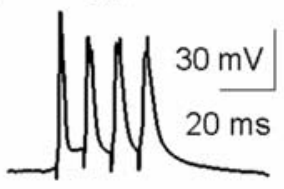

C

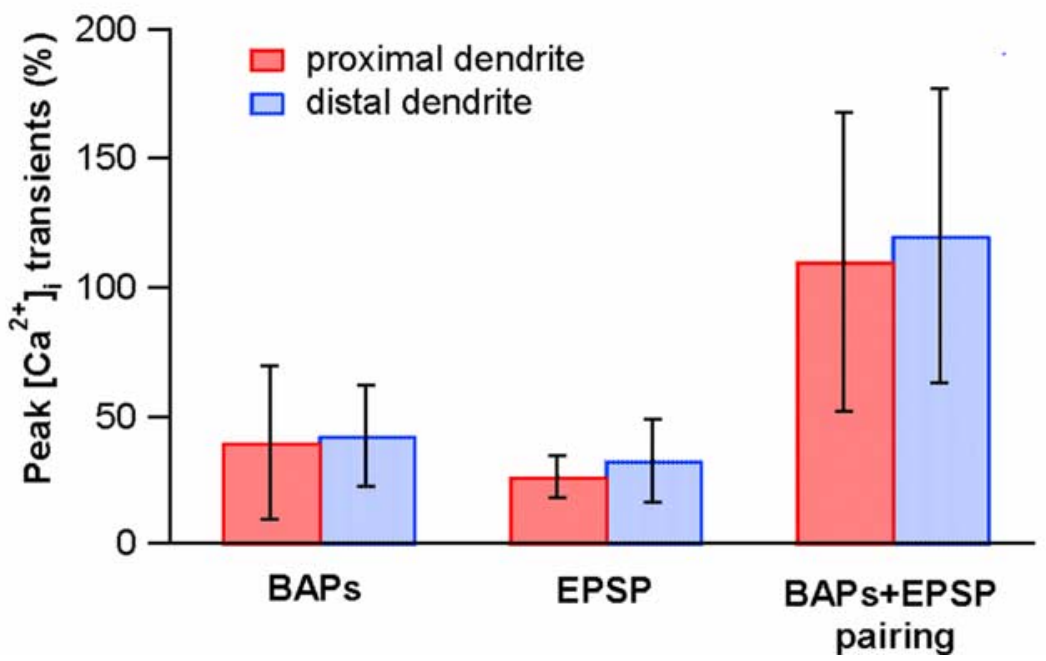

Figure 2. Calcium transients in proximal and distal basal dendrites. $A$, Experimental setup. A basal branch of a layer 5 pyramidal neuron loaded with 0GB-6 (1 mM). Calcium imaging was performed from a proximal (red) and a distal (blue) region. Calcium imaging measurements were collected from dendritic spines both in the proximal region (dotted red line) and the distal region (dotted blue line). $\boldsymbol{B}$, Calcium transients presented as $\Delta F / F(\%)$ were evoked for proximal (red) and distal (blue) regions by local EPSPs (bottom left), a train of four BAPs (bottom middle), and pairing of a train of four BAPs with local EPSPs (bottom right). C, A summary plot of the peak calcium transients at proximal and distal regions evoked by a train of BAPs, EPSP, and pairing of a train of BAPs and EPSP (as in the induction protocol) is presented. All measurements were done with 0GB-6 (0.5-1 $\mathrm{mm})$. Note that no significant difference in the calcium transients was observed between proximal and distal regions in all stimulus paradigms used ( $p$ values were not significant for all three comparisons). Error bars represent SD. 
A

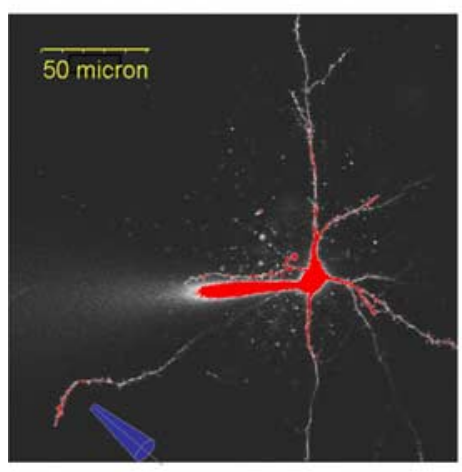

B

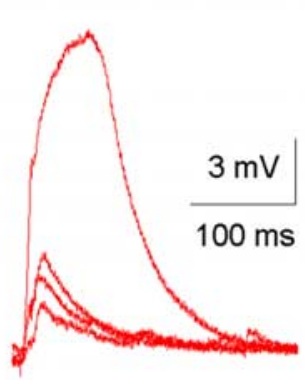

C

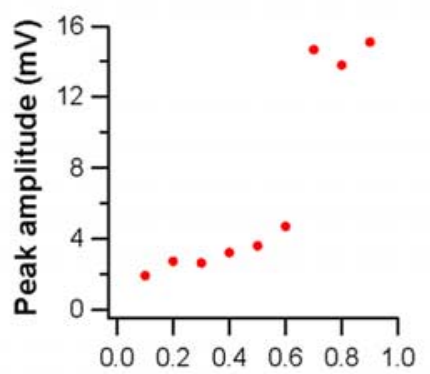

Stimulus intensity (mA)

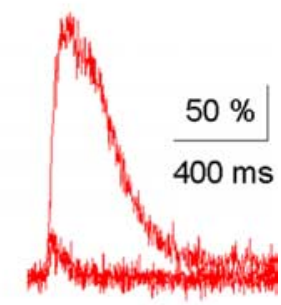

D

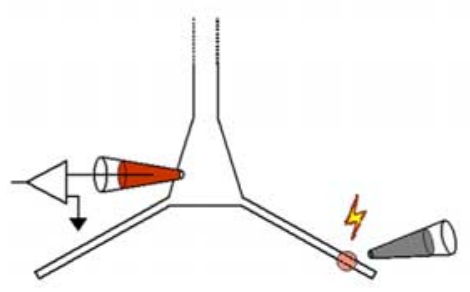

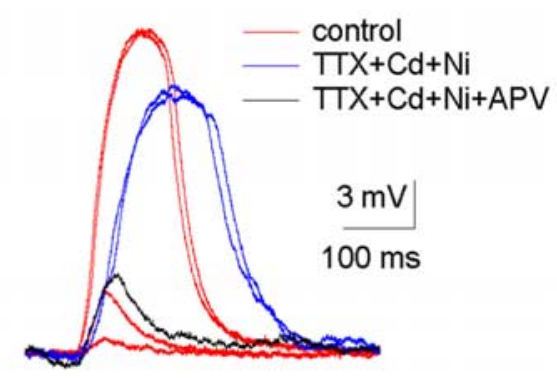

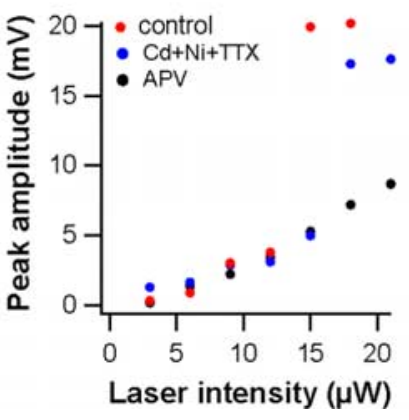

Figure 3. NMDA spikes in basal dendrites of layer 2-3 pyramidal neurons. $\boldsymbol{A}$, Experimental set up: a layer 2-3 pyramidal neuron was loaded with 0 GB-6 (500 $\mu \mathrm{m}$ ), and a theta-stimulating electrode (shown schematically) was placed close to a distal region ( $120 \mu \mathrm{m}$ from the soma). $\boldsymbol{B}$, Postsynaptic voltage responses evoked by two stimuli at $100 \mathrm{~Hz}$ are shown for different stimulus intensities. The stimulus intensity was increased gradually until an all-or-none local spike was initiated. The peaks of the postsynaptic voltage responses are plotted as a function of the synaptic stimulus intensity (right). C, Calcium transients obtained from an activated dendritic spine evoked by a local dendritic spike, a burst of four action potentials ( $100 \mathrm{~Hz}$ ), and an EPSP ( $2 \mathrm{mV}$ at the soma). D, NMDA spikes evoked by UV-laser uncaging of glutamate (Left, experimental set up shown schematically). Caged glutamate (MNI-glutamate, 5 mm) was delivered by pressure ejection locally through an electrode ( $2 \mu \mathrm{m}$ tip diameter, $5 \mathrm{mbar}$ ). Middle, Single traces of the postsynaptic voltage response evoked by glutamate uncaging at increasing laser intensities in control conditions (red) and after addition of TTX $(1 \mu \mathrm{m})$, cadmium (100 $\mu \mathrm{m})$, and nickel (100 $\mu \mathrm{m})$ (blue). Adding APV to the cocktail of blockers (50 $\mu \mathrm{m}$; black) blocked the spike. Right, The peak postsynaptic amplitude is plotted as a function of the laser intensities for control conditions, in the presence of TTX, cadmium, and nickel (red) and in the presence of TTX, cadmium, nickel, and APV (black).

After establishing that basal dendrites of layer 2-3 pyramidal neurons also support the initiation of local NMDA spikes, we next investigated the possible role of NMDA spikes in the induction of LTP in both layers 5 and 2-3 basal dendrites. Surprisingly, pairing of EPSPs with NMDA spikes evoked by two focal theta electrodes 30-40 $\mu \mathrm{m}$ apart (typically $15-30$ repetitions at $0.1 \mathrm{~Hz}$, and four neurons with 60 repetitions) did not evoke robust LTP in both layer 2-3 and layer 5 pyramidal neurons (Fig. 4). On average, the EPSP amplitude $40 \mathrm{~min}$ after induction was $102 \pm$ $44 \%$ of the preinduction control value (48 dendrites, 28 layer 2-3, 20 layer 5; no significant difference between layer 5 and layer 2-3 neurons). When we used a small number of repetitions in the induction protocol (one to five only), no change was observed in the EPSP amplitude, in contrast to the report by Holthoff et al. (2004). The difference between our findings and those reported by Holthoff et al. (2004) may result from differences in the experimental conditions including animal species (rats vs mice), brain area (somatosensory vs visual), and type of dendrites tested (basal vs a mixture of apical, oblique, and basal).

The experiments involved with NMDA spikes were performed only on the distal half of basal branches, because we were unable to reliably obtain synaptically evoked NMDA spikes in proximal basal dendrites, despite our ability to stimulate these regions focally. Glutamate uncaging and modeling studies indicate that initiation of NMDA spikes in proximal basal regions requires much larger currents compared with distal regions (A. Polsky and J. Schiller, unpublished observations). We speculate that these current requirements were not satisfied in our experiments.

Our data show that, despite the fact that NMDA spikes were accompanied by very large calcium transients (Fig. $3 C$ ), they did not cause LTP. We raised the possibility that calcium influx evoked by NMDA spikes was too high to induce LTP. To examine this possibility, we attempted to induce LTP by pairing EPSPs and NMDA spikes in the presence of 4-10 mM intracellular BAPTA. Despite partially buffering the intracellular calcium concentrations, no long-term changes in the average EPSP were observed. Forty minutes after induction in the presence of intracellular BAPTA, the average EPSP amplitude reached $100 \pm 52 \%$ of the preinduction control EPSP amplitude $[n=15$ in eight neurons; no significant differences between layer $2-3(n=9)$ and layer 5 neurons $(n=6)]$.

Previous studies reported that the NR2B subunits of the NMDA receptor mediate LTD rather than LTP (Liu et al., 2004; Massey et al., 2004). We raised the possibility that during generation of NMDA spikes the NR2B subtype of NMDA-receptor subunits are preferentially activated and, hence, preclude induction of LTP. However, the selective NR2B subunit blocker ifenprodil $(3 \mu \mathrm{M})$ reduced the amplitude of the basal spikes by $<10 \%(n=4)$. Moreover, we failed to 
induce LTP by pairing of NMDA spikes and distal EPSPs in the presence of ifenprodil $(n=3)$.

\section{BDNF and NMDA spikes can mediate LTP in distal basal dendrites}

Together, our findings indicate that basal dendrites contain two separate compartments with respect to the ability to undergo LTP. The first compartment, located at the proximal half of the basal tree, contains synapses that can undergo plasticity in response to the global suprathreshold activity of the neuron using pairing protocols. The second compartment, located at the distal half of the basal tree, contains synapses that are resistant to plasticity changes by either global BAPs or local dendritic spikes.

In light of these findings, we next investigated whether distal synapses still retain their ability to undergo LTP under more special conditions. More specifically, we examined whether these synapses can undergo LTP when a neuromodulatory molecule is present during the induction period. Because BDNF is a potent neurotrophic factor that modulates gating of NMDA-receptor channels (Levine and Kolb, 2000), and also enhances long-term plasticity (Korte et al., 1995; Kang et al., 1997; Black, 1999; Minichiello et al., 1999; Schuman, 1999; Kovalchuk et al., 2002; Du and Poo, 2004), we investigated its effect on plasticity of distal basal synapses.

Pairing of BDNF with NMDA spikes and local EPSPs at the same dendritic segment caused a large and robust LTP (Fig. 5). On average, pairing of EPSPs and NMDA spikes (15 times at 0.1 $\mathrm{Hz}$ ) in the presence of locally applied BDNF resulted in a $319 \pm$ $116 \%$ increase of the EPSP amplitude, compared with its control value ( $n=16,10$ layer 2-3, 6 layer 5; no significant difference between layer 5 and layer 2-3 neurons). In fact, five repetitions of paired NMDA spikes and EPSPs $(0.1 \mathrm{~Hz})$ were sufficient to induce LTP in the presence of BDNF $(n=4)$.

The effect of BDNF on LTP was mediated by the receptor tyrosine kinase TrkB pathway. Pretreatment of the slice for 10 min with the tyrosine kinase antagonist K252a (150 nM) markedly reduced the extent of LTP evoked by the pairing of EPSPs with NMDA spikes and BDNF. In all experiments, NMDA spikes were not affected by K252a. In the presence of K252a, the average EPSP amplitude measured 40 min after induction increased by only $154 \pm 49 \%(n=4)$, compared with $319 \pm 116 \%$ without the tyrosine kinase blocker $(p=0.02)$.

Induction of LTP required the initiation of NMDA spikes. When EPSPs rather than NMDA spikes were paired with BDNF, no long-term synaptic plasticity was induced and the amplitude of EPSPs remained unchanged (Fig. $5 E)(n=4)$. Application of BDNF on inactive synapses also had no long-term effects on the EPSP amplitude $(n=3)$. In addition, when we activated only the NMDA-spike site (in the presence of BDNF) while the second electrode at the EPSP site $(30-40 \mu \mathrm{m}$ apart) was not activated during the induction protocol, no potentiation of the unactivated EPSP site was observed $(n=5)$.

We next investigated whether distal synapses can undergo LTP using pairing of EPSPs and BAPs in the presence of BDNF.
B

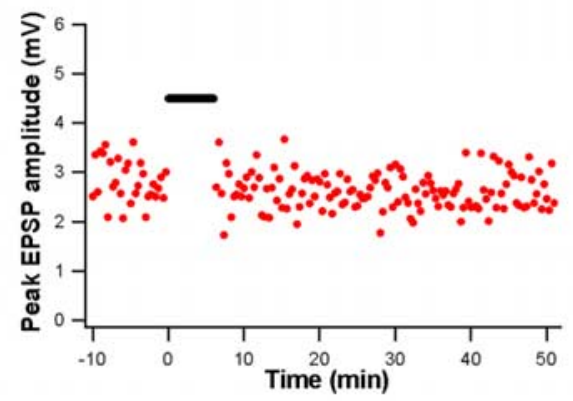

D

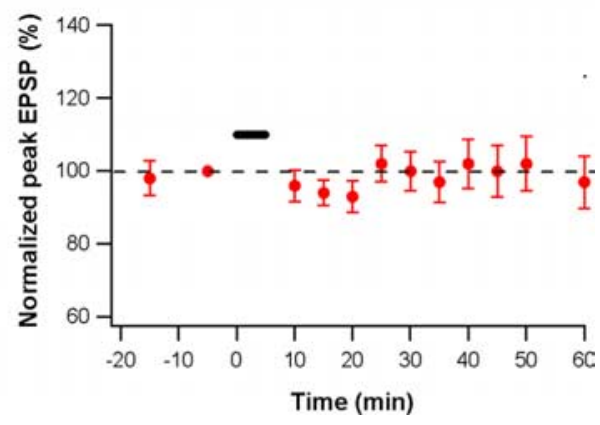


A

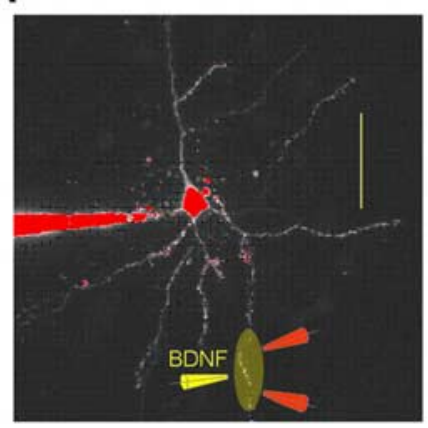

C

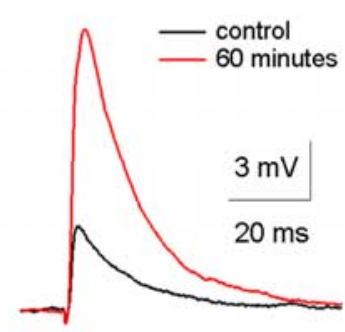

B

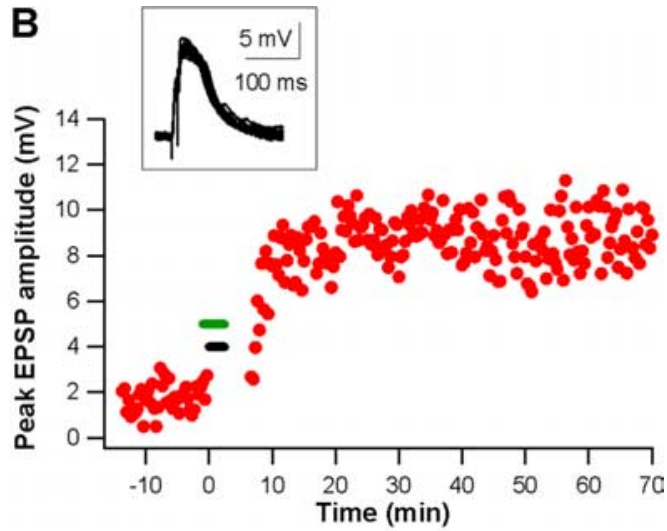

D

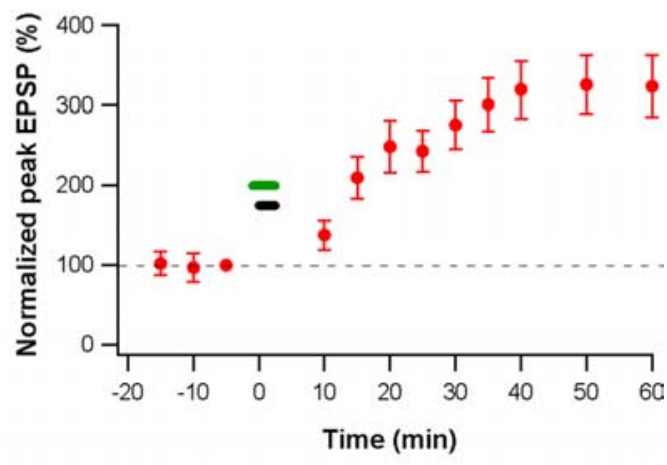

E

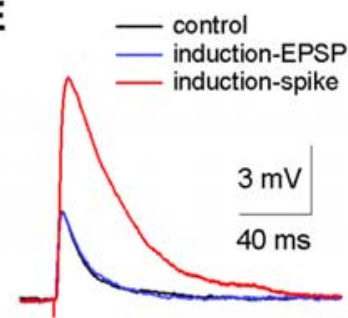

Figure 5. Pairing of NMDA spikes and BDNF is necessary for LTP induction in distal basal dendritic regions. $A$, Experimental setting. A layer 2-3 pyramidal neuron was loaded with OGB-1 $(200 \mu \mathrm{m})$. Two stimulating electrodes (red) and a third electrode used to deliver BDNF (yellow; $50 \mathrm{ng} / \mathrm{ml}$ ) were positioned near a basal dendrite. $B$, Peak EPSP amplitude before and after the induction protocol (black, voltage stimulation; green, BDNF application). The inset shows the induction protocol. NMDA spikes $(15,0.1 \mathrm{~Hz})$ were delivered concomitant to local BDNF injection. C, A comparison of an average EPSP before the induction (10 min; black), after the induction (60 min; red), and in the presence of APV (blue) is shown. Note the large increase in the AMPA component. $\boldsymbol{D}$, A summary plot of a normalized average of 16 similar experiments shows a large potentiation after this induction protocol $(319 \pm 116 \%)$. $E$, As a control to the requirement of NMDA spikes, the same protocol was repeated, but instead of evoking spikes, only local EPSPs were evoked (black, control; blue, 40 min after EPSP induction). Later, in the same dendrite, a second induction was performed with local NMDA spikes (red trace; 40 min after induction). The cell in $B, C$ is different from the cell in $\boldsymbol{E}$. Error bars represent SEM.

jnowski, 2000; Sjostrom et al., 2001, 2002; Dan and Poo, 2004). We addressed the temporal rules governing NMDA spikemediated LTP. In these experiments, we varied the time delay between NMDA spikes and the paired EPSPs evoked by a second electrode located $30-40 \mu \mathrm{m}$ along the same branch. The BDNF was locally applied throughout the induction protocol period. NMDA spike-mediated LTP showed a dramatically different temporal window than STDP (Fig. 7). First, only LTP and not LTD was evoked. Second, NMDA spikes opened a prolonged time window for plasticity, lasting $\sim 150 \mathrm{~ms}$. Third, LTP was induced regardless of whether spikes preceded or followed EPSPs. However, the potentiation time window was asymmetrical and preferred postsynaptic before presynaptic activation order at a ratio of 5:1 (Fig. 7C).

\section{Discussion}

In this study, we report that the basal tree is composed of proximal and distal plasticity compartments. Inputs in the proximal compartment undergo plasticity using conventional pairing protocols. In contrast, inputs in the distal plasticity compartment are relatively resistant to change and require unusual conditions to do so. These synapses undergo LTP only when NMDA spikes are combined with a gating molecule (BDNF). It is possible that plasticity rules change gradually between the proximal and distal compartments with no sharp borders between the two compartments. Additional experiments with finer spatial resolution of focal activation will be needed to clarify this point.

Location-dependent plasticity rules were reported previously for apical dendrites of layers 2-3 and layer 5 pyramidal neurons (Froemke et al., 2005; Sjostrom and Hausser, 2006). Our findings indicate that plasticity compartments markedly differ between basal and apical dendrites. In apical dendrites of layers 2-3 pyramidal neurons, proximal and distal inputs can undergo LTP when paired with BAPs and differences between proximal and distal inputs are mainly manifested in a slight amplitude reduction of LTP (Froemke et al., 2005). In addition, it was shown previously that distal apical dendrites of layer 5 pyramidal neurons undergo LTD and not LTP in response to STDP induction protocols (Letzkus et al., 2006; Sjostrom and Hausser, 2006). It is important to note that distal apical dendrites of both layer 5 and CA1 pyramidal neurons undergo LTP in response to the local spikes within the dendritic branch (Golding et al., 2002; Lisman and Spruston, 2005) (J. Schiller, unpublished observations regarding apical dendrites of layer 5 pyramidal neurons). In contrast to apical dendrites, inputs innervating distal basal dendrites fail to undergo LTP when paired with either BAPs or local spikes within the branch.

The significance of functional dendritic compartmentalization with respect to plasticity and integration depends on the spatial dendritic innervation pattern (Hausser and Mel, 2003). Previous studies have reported spatial segregation of inputs carrying different information into different dendritic regions (Single and Borst, 1998; Megias et al., 2001; Euler et al., 2002). For example, feedback corticocortical inputs terminate on the tuft apical branches (Pandya and Yeterian, 1985; Cauller and Connors, 1994). Also, in basal dendrites, some evidence supports spatial dendritic segregation of inputs according to the information they carry. Inputs originating from within the same cortical column preferentially innervate proximal basal dendrites (Markram et al., 1997a; Feldmeyer et al., 2002). Interestingly, noradrenergic innervation may also differ along the basal dendritic tree (Benavides-Piccione et al. 
A

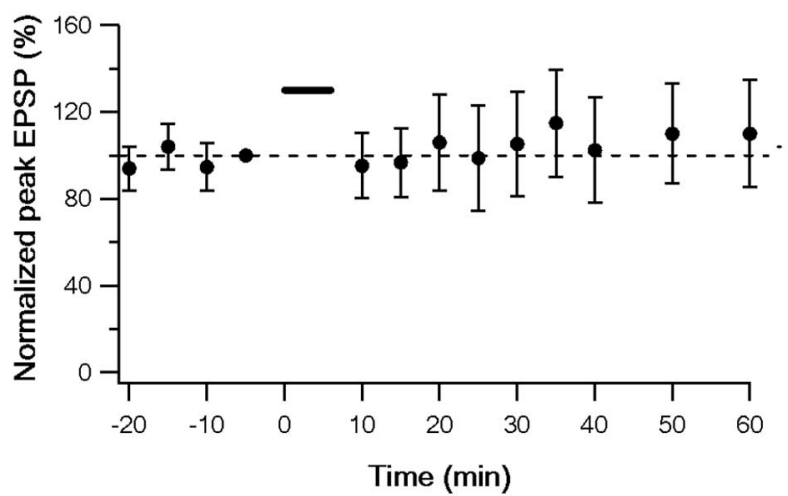

B
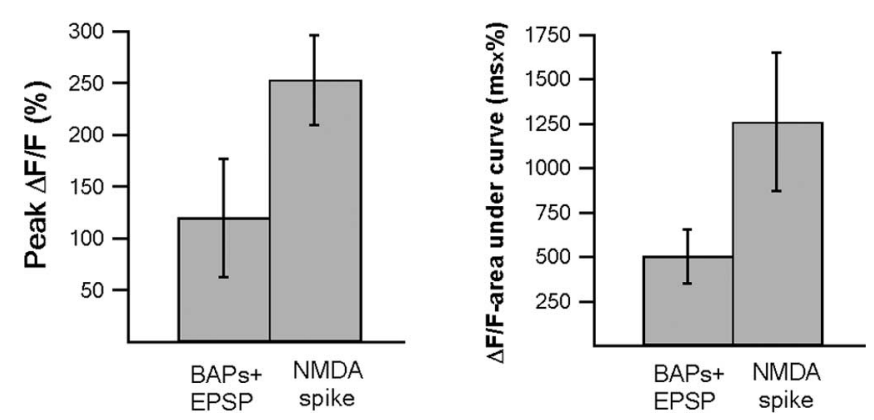

Figure 6. Pairing protocol in the presence of BDNF did not induce LTP in distal basal dendrites. $\boldsymbol{A}$, Pairing BAPs with EPSPs (10 ms delay) in the presence of BDNF did not induce LTP in distal basal dendrites. The pairing protocol consisted of a burst of BAPs (4 BAPs; $100-150 \mathrm{~Hz}$ ), delivered $10 \mathrm{~ms}$ after a single EPSP in the presence of local application of BDNF. A summary plot of a normalized average of 12 experiments using this protocol is shown. Error bars indicate SEM. $\boldsymbol{B}$, Comparison of the averaged (mean \pm SD) peak calcium transients (left) and area under the curve (right) evoked by pairing of BAPs and EPSP and NMDA spikes.

2005). Thus, it is possible that the ability of different anatomical pathways to undergo LTP will markedly differ depending on the dendritic plasticity compartment on which they terminate.

In this study, we describe a special case of synapses that require the presence of both NMDA spikes and the neuromodulatory molecule BDNF to undergo LTP. The involvement of the neurotrophic factor BDNF has been well documented in a variety of developmental processes such as survival and differentiation (Binder and Scharfman, 2004) as well as synaptic plasticity (Korte et al., 1995; Black, 1999; Minichiello et al., 1999; Schuman, 1999; Schinder and Poo, 2000). Previous studies have shown that the addition of exogenous BDNF can enhance plasticity (Kovalchuk et al., 2002; Du and Poo, 2004). Moreover, genetic deletion of the BDNF or TrkB genes impaired the ability of synapses to undergo LTP in the hippocampus, hence, demonstrating the physiological role of endogenous BDNF in LTP induction (Korte et al., 1995, 1996; Kang et al., 1997; Bramham and Messaoudi, 2005). It is interesting to note that, in contrast to previous studies, the BDNF in the case of distal basal synapses acts as an essential "gating" molecule and not just as a facilitatory molecule as shown for other pathways (Schuman, 1999).

One of the main questions raised by our findings is what physiological conditions will promote the release of BDNF in distal synapses? The factors that control the release of BDNF in the brain in vivo are not yet clear. Previous studies have shown release of BDNF from both axons and dendrites (Hartmann et al., 2001; Kohara et al., 2001) in an activity-dependent manner (Bonhoef-
A

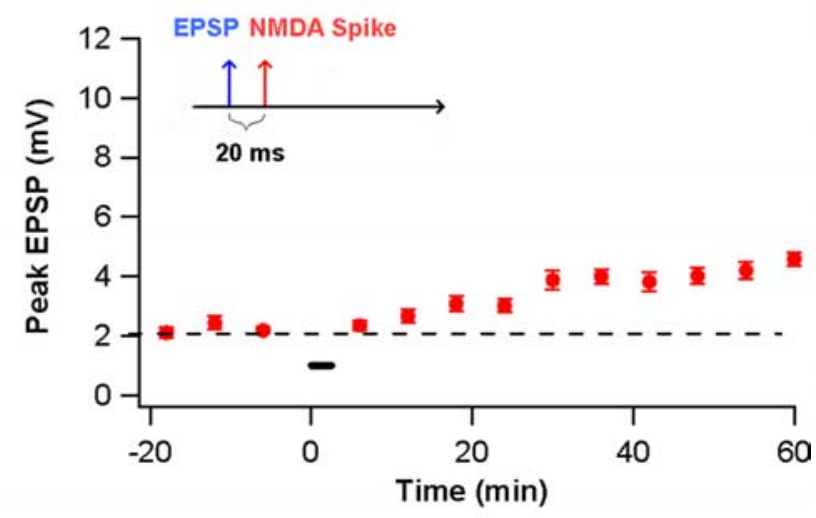

B

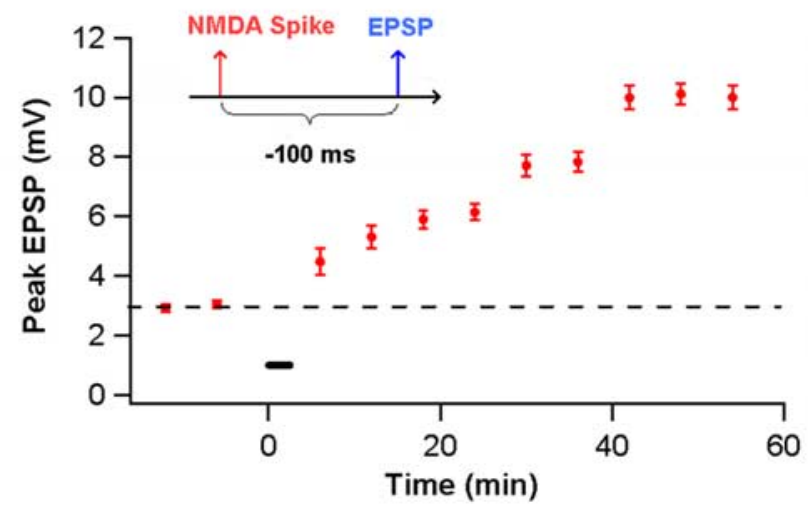

C

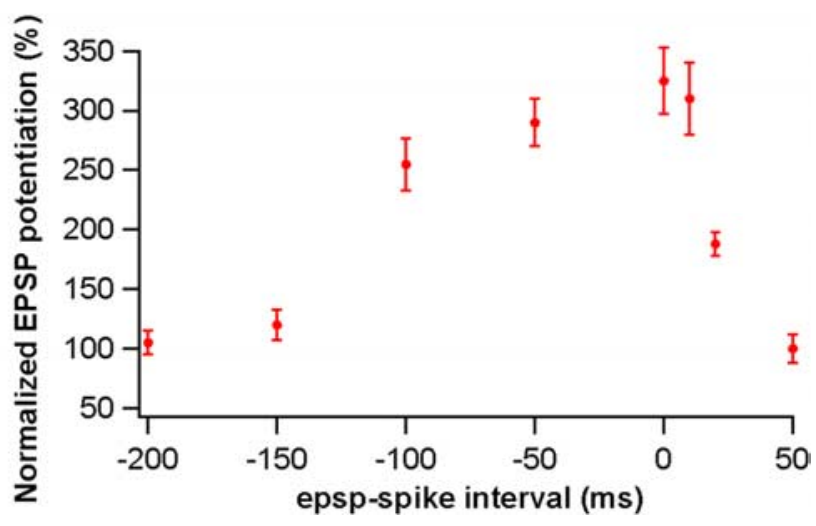

Figure 7. A large time window for plasticity after induction with NMDA spikes. Two stimulating electrodes were positioned ( $40 \mu \mathrm{m}$ apart) at a basal dendrite. The time delay between an EPSP evoked with one electrode and a spike evoked with a second electrode was changed from $50 \mathrm{~ms}$ (EPSP before spike) to $-200 \mathrm{~ms}$ (EPSP after spike). All inductions were performed in the presence of BDNF delivered locally with a third electrode. $A$, Example experiment presenting average peak in control and after induction protocol using a time delay of $20 \mathrm{~ms}$ (inset, EPSP before spike). $\boldsymbol{B}$, Same as $\boldsymbol{A}$, except that the time delay was $-100 \mathrm{~ms}$ (inset, EPSP after spike). C, Normalized average summary before and after induction at delays from $50 \mathrm{~ms}$ to $-200 \mathrm{~ms}$ from 42 experiments. Note the large time window of $\sim 150$ ms opened by the NMDA spike for plasticity. Error bars represent SEM.

fer, 1996; Rocamora et al., 1996; Altar and DiStefano, 1998; Fawcett et al., 1998; Kohara et al., 2001; Kojima et al., 2001; Lessmann et al., 2003; Aicardi et al., 2004; Adachi et al., 2005). Our results show that the induction protocols we used in the slice preparation were not efficient in releasing BDNF to distal basal synapses. It is possible that under in vivo conditions, other activation pat- 
terns or activation of additional pathways will supply a sufficient amount of endogenous BDNF that, when paired with NMDA spikes, will result in LTP induction in distal synapses.

The mechanism by which the combined NMDA spikes and BDNF exert their effects in our case is not known. It has been reported previously that BDNF acts postsynaptically by depolarizing dendrites and evoking local calcium transients in dendritic spines (Blum et al., 2002; Kovalchuk et al., 2002). In our case, the signal required for LTP induction was the NMDA spike, which to begin with is a large local voltage signal introducing very a large calcium influx postsynaptically. Thus, it is unlikely that the mechanism by which BDNF acts is by adding postsynaptic depolarization or increasing the calcium influx. Rather, we hypothesize that BDNF acts on one or more processes of the postcalcium biochemical cascade leading to LTP (Huang and Reichardt, 2003). In addition, the mechanisms underlying the preference of NMDA spikes over BAPs in LTP induction at distal synapses are not known. NMDA spikes are associated with a much larger activation of NMDA current compared with EPSPs or BAPs. Induction of LTP by NMDA spikes may result from the larger calcium influx evoked by NMDA spikes, or by more effective activation of NMDA receptor-linked biochemical cascades. In addition, NMDA spikes may be more efficient in promoting secondary activation of mGluRs compared with BAPs. Additional studies are required to differentiate between these different possibilities.

Presently, it is difficult to determine the physiological role of the distal plasticity compartment. It is possible that the special conditions required for LTP in distal basal dendrites are not fulfilled in vivo and inputs terminating on distal basal dendrites serve to carry hard-wired information that is protected from activity-dependent changes. If, however, NMDA-spike mediated LTP occurs in vivo, it can potentially serve three main roles.

First, the temporal plasticity rules used when NMDA spikes serve as the postsynaptic signal markedly differ compared with STDP temporal rules. The potentiation time window of NMDA spike-mediated LTP are much longer than that of STDP lasting $\sim 150 \mathrm{~ms}$ and, in contrast to STDP, a reversed pre/post activation order is preferred. The temporal rules of STDP are well suited to mediate plasticity of feedforward inputs. In contrast, the plasticity rules that apply to feedback synapses are expected to have different properties: they are expected to be "noncausal," because feedback inputs will normally fire sometime after the neuron was activated. Moreover, the timing requirements for the induction of plastic changes should be much broader than for the feedforward pathway, given that feedback pathways are polysynaptic with varying numbers of intermediate neurons. The NMDA spike-mediated LTP provides all of these requirements; thus, it is well suited to potentiate feedback inputs. An interesting, yet speculative possibility is that feedback pathways preferentially terminate unto distal basal dendrites and, hence, are governed by a different set of plasticity rules.

A second possible role is in developmental wiring of the cortical network. Previous studies have shown that synaptic plasticity is age dependent. Certain pathways, most notably the thalamocortical fibers, lose their ability to undergo long-term synaptic plasticity as the animal matures (Crair and Malenka, 1995; Kirkwood et al., 1995), whereas other pathways require stronger postsynaptic signals for the induction of LTP with maturation (Pike at al., 1999). Our study was performed on juvenile (17-24 d of age) rats. It is possible that different plasticity rules serve to wire different anatomical pathways or that maturation of the plasticity rules of inputs depends on their dendritic location, with distal inputs maturing earlier. Additional studies are re- quired to establish whether distal inputs can undergo plasticity with STDP rules in younger animals and whether proximal inputs require more intense activation to undergo plasticity in older animals.

A third potential physiological role of the NMDA spikemediated LTP is to increase the stability of previously acquired information. Synapses located in the distal plasticity compartment are unlikely to change accidentally as a result of the ongoing network activity, because they require both the initiation of a local spike and the release of BDNF. The existence of different plasticity compartments with diverse plasticity rules may serve to increase the processing capabilities of the network (Bastian et al., 2004).

\section{References}

Adachi N, Kohara K, Tsumoto T (2005) Difference in trafficking of brainderived neurotrophic factor between axons and dendrites of cortical neurons, revealed by live-cell imaging. BMC Neurosci 6:42.

Aicardi G, Argilli E, Cappello S, Santi S, Riccio M, Thoenen H, Canossa M (2004) Induction of long-term potentiation and depression is reflected by corresponding changes in secretion of endogenous brain-derived neurotrophic factor. Proc Natl Acad Sci USA 101:15788-15792.

Altar CA, DiStefano PS (1998) Neurotrophin trafficking by anterograde transport. Trends Neurosci 10:433-437.

Artola A, Singer W (1993) Long-term depression of excitatory synaptic transmission and its relationship to long-term potentiation. Trends Neurosci 16:480-487.

Bastian J, Chacron MJ, Maler L (2004) Plastic and nonplastic pyramidal cells perform unique roles in a network capable of adaptive redundancy reduction. Neuron 41:767-779.

Bear MF, Kirkwood A (1993) Neocortical long-term potentiation. Curr Opin Neurobiol 3:197-202.

Benavides-Piccione R, Arellano JI, DeFelipe J (2005) Catecholaminergic innervation of pyramidal neurons in the human temporal cortex. Cereb Cortex 10:1584-1591.

Bi GQ, Poo MM (1998) Synaptic modifications in cultured hippocampal neurons: dependence on spike timing, synaptic strength, and postsynaptic cell type. J Neurosci 18:10464-10472.

Binder DK, Scharfman HE (2004) Brain-derived neurotrophic factor. Growth Factors 22:123-131.

Black IB (1999) Trophic regulation of synaptic plasticity. J Neurobiol 41:108-118.

Bliss TVP, Collingridge GL (1993) A synaptic model of memory: long term potentiation in the hippocampus. Nature 361:31-39.

Blum R, Kafitz KW, Konnerth A (2002) Neurotrophin-evoked depolarization requires the sodium channel $\mathrm{Na}(\mathrm{V}) 1.9$. Nature 419:687-693.

Bonhoeffer T (1996) Neurotrophins and activity-dependent development of the cortex. Curr Opin Neurobiol 6:119-126.

Bramham CR, Messaoudi E (2005) BDNF function in adult synaptic plasticity: the synaptic consolidation hypothesis. Prog Neurobiol 76:99-125.

Cauller LJ, Connors BW (1994) Synaptic physiology of horizontal afferents to layer I in slices of rat SI neocortex. J Neurosci 14:751-762.

Crair MC, Malenka RC (1995) A critical period for long-term potentiation at thalamocortical synapses. Nature 375:325-328.

Dan Y, Poo MM (2004) Spike timing-dependent plasticity of neural circuits. Neuron 44:23-30.

Du JL, Poo MM (2004) Rapid BDNF-induced retrograde synaptic modification in a developing retinotectal system. Nature 429:878-883.

Euler T, Detwiler PB, Denk W (2002) Directionally selective calcium signals in dendrites of starburst amacrine cells. Nature 418:845-852.

Fawcett JP, Bamji SX, Causing CG, Aloyz R, Ase AR, Reader TA, McLean JH, Miller FD (1998) Functional evidence that BDNF is an anterograde neuronal trophic factor in the CNS. J Neurosci 18:2808-2821.

Feldman DE (2000) Timing-based LTP and LTD at vertical inputs to layer II/III pyramidal cells in rat barrel cortex. Neuron 27:45-56.

Feldmeyer D, Lubke J, Silver RA, Sakmann B (2002) Synaptic connections between layer 4 spiny neurone-layer $2 / 3$ pyramidal cell pairs in juvenile rat barrel cortex: physiology and anatomy of interlaminar signaling within a cortical column. J Physiol (Lond) 538:803-822.

Franks KM, Sejnowski TJ (2002) Complexity of calcium signaling in synaptic spines. Bioessays 24:1130-1144. 
Froemke RC, Poo MM, Dan Y (2005) Spike-timing-dependent synaptic plasticity depends on dendritic location. Nature 434:221-225.

Golding NL, Staff NP, Spruston N (2002) Dendritic spikes as a mechanism for cooperative long-term potentiation. Nature 418:326-331.

Hartmann M, Heumann R, Lessmann V (2001) Synaptic secretion of BDNF after high-frequency stimulation of glutamatergic synapses. EMBO J 20:5887-5897.

Hausser M, Mel B (2003) Dendrites: bug or feature? Curr Opin Neurobiol 13:372-383.

Holthoff K, Kovalchuk Y, Yuste R, Konnerth A (2004) Single-shock LTD by local dendritic spikes in pyramidal neurons of mouse visual cortex. J Physiol (Lond) 560:27-36.

Huang EJ, Reichardt LF (2003) Trk receptors: roles in neuronal signal transduction. Annu Rev Biochem 72:609-642.

Johnston D, Hoffman DA, Magee JC, Poolos NP, Watanabe S, Colbert CM, Migliore M (2000) Dendritic potassium channels in hippocampal pyramidal neurons. J Physiol (Lond) 525:75-81.

Kang H, Welcher AA, Shelton D, Schuman EM (1997) Neurotrophins and time: different roles for TrkB signaling in hippocampal long-term potentiation. Neuron 19:653-664.

Kirkwood A, Lee HK, Bear MF (1995) Co-regulation of long-term potentiation and experience-dependent synaptic plasticity in visual cortex by age and experience. Nature 375:328-331.

Koester HJ, Sakmann B (1998) Calcium dynamics in single spines during coincident pre- and postsynaptic activity on relative timing of backpropagating action potentials and subthreshold excitatory postsynaptic potentials. Proc Natl Acad Sci USA 95:9596-9601.

Kohara K, Kitamura A, Morishima M, Tsumoto T (2001) Activitydependent transfer of brain-derived neurotrophic factor to postsynaptic neurons. Science 291:2419-2423.

Kojima M, Takei N, Numakawa T, Ishikawa Y, Suzuki S, Matsumoto T, Katoh-Semba R, Nawa H, Hatanaka H (2001) Biological characterization and optical imaging of brain-derived neurotrophic factor-green fluorescent protein suggest an activity-dependent local release of brainderived neurotrophic factor in neurites of cultured hippocampal neurons. J Neurosci Res 64:1-10.

Korngreen A, Sakmann B (2000) Voltage-gated $\mathrm{K}^{+}$channels in layer 5 neocortical pyramidal neurones from young rats: subtypes and gradients. J Physiol (Lond) 525:621-639.

Korte M, Carroll P, Wolf E, Brem G, Thoenen H, Bonhoeffer T (1995) Hippocampal long-term potentiation is impaired in mice lacking brainderived neurotrophic factor. Proc Natl Acad Sci USA 92:8856-8860.

Korte M, Griesbeck O, Gravel C, Carroll P, Staiger V, Thoenen H, Bonhoeffer $\mathrm{T}$ (1996) Virus-mediated gene transfer into hippocampal CA1 region restores long-term potentiation in brain-derived neurotrophic factor mutant mice. Proc Natl Acad Sci USA 93:12547-12552.

Kovalchuk Y, Hanse E, Kafitz KW, Konnorth A (2002) Postsynaptic induction of BDNF-mediated long-term potentiation. Science 295:1729-1734.

Larkman AU (1991) Dendritic morphology of pyramidal neurones of the visual cortex of the rat: III. Spine distributions. J Comp Neurol 306:332-343.

Larkum ME, Zhu JJ, Sakmann B (1999a) A new cellular mechanism for coupling inputs arriving at different cortical layers. Nature 398:338-341.

Larkum ME, Kaiser KM, Sakmann B (1999b) Calcium electrogenesis in distal apical dendrites of layer 5 pyramidal cells at a critical frequency of back-propagating action potentials Proc Natl Acad Sci USA 96:14600-14604.

Lessmann V, Gottmann K, Malcangio M (2003) Neurotrophin secretion: current facts and future prospects. Prog Neurobiol 69:341-374.

Letzkus JJ, Kampa BM, Stuart GJ (2006) Learning rules for spike timingdependent plasticity depend on dendritic synapse location. J Neurosci 11:10420-10429.

Levine ES, Kolb JE (2000) Brain-derived neurotrophic factor increases activity of NR2B-containing $\mathrm{N}$-methyl-D-aspartate receptors in excised patches from hippocampal neurons. J Neurosci Res 62:357-362.

Lisman J, Spruston N (2005) Postsynaptic depolarization requirements for
LTP and LTD: a critique of spike timing dependent plasticity. Nat Neurosci 8:839-841.

Liu L, Wong TP, Pozza MF, Lingenhoehl K, Wang Y, Sheng M, Auberson YP, Wang YT (2004) Role of NMDA receptor subtypes in governing the direction of hippocampal synaptic plasticity. Science 304:1021-1024.

Magee JC (1999) Dendritic Ih normalizes temporal summation in hippocampal CA1 neurons. Nat Neurosci 2:848.

Magee JC (2000) Dendritic integration of excitatory synaptic input. Nat Rev Neurosci 1:181-190.

Markram H, Lubke J, Frotscher M, Roth A, Sakmann B (1997a) Physiology and anatomy of synaptic connections between thick tufted pyramidal neurones in the developing rat neocortex. J Physiol (Lond) 500:409-440.

Markram H, Lubke J, Frotscher M, Sakmann B (1997b) Regulation of synaptic efficacy by coincidence of postsynaptic APs and EPSPs. Science 275:213-215.

Massey PV, Johnson BE, Moult PR, Auberson YP, Brown MW, Molnar E, Collingridge GL, Bashir ZI (2004) Differential roles of NR2A and NR2B-containing NMDA receptors in cortical long-term potentiation and long-term depression. J Neurosci 8:7821-7828.

Megias M, Emri Z, Freund TF, Gulyas AI (2001) Total number and distribution of inhibitory and excitatory synapses on hippocampal CA1 pyramidal cells. Neuroscience 102:527-540.

Migliore M, Hoffman DA, Magee JC, Johnston D (1999) Role of an A-type $\mathrm{K}^{+}$conductance in the back-propagation of action potentials in the dendrites of hippocampal pyramidal neurons. J Comput Neurosci 7:5-15.

Minichiello L, Korte M, Wolfer D, Kuhn R, Unsicker K, Cestari V, RossiArnaud C, Lipp HP, Bonhoeffer T, Klein R (1999) Essential role for TrkB receptors in hippocampus-mediated learning. Neuron 24:401-414.

Pandya DN, Yeterian EH (1985) Architecture and connections of cortical association areas. In: Cerebral cortex, Vol IV (Peters A, Jones EG, eds), pp 3-61. New York: Plenum.

Paulsen O, Sejnowski TJ (2000) Natural patterns of activity and long-term synaptic plasticity. Curr Opin Neurobiol 10:172-179.

Pike FG, Meredith RM, Olding AW, Paulsen O (1999) Rapid report: postsynaptic bursting is essential for "Hebbian" induction of associative long-term potentiation at excitatory synapses in rat hippocampus. J Physiol (Lond) 518:571-576.

Polsky A, Mel BW, Schiller J (2004) Computational subunits in thin dendrites of pyramidal neurons. Nat Neurosci 7:621-627.

Rocamora N, Welker E, Pascual M, Soriano E (1996) Upregulation of BDNF mRNA expression in the barrel cortex of adult mice after sensory stimulation. J Neurosci 16:4411-4419.

Schiller J, Schiller Y, Stuart G, Sakmann B (1997) Calcium action potentials restricted to distal apical dendrites of rat neocortical pyramidal neurons. J Physiol (Lond) 505:605-616.

Schiller J, Major G, Koester HJ, Schiller Y (2000) NMDA spikes in basal dendrites of cortical pyramidal neurons. Nature 404:285-289.

Schinder AF, Poo M (2000) The neurotrophin hypothesis for synaptic plasticity. Trends Neurosci 23:639-645.

Schuman EM (1999) Neurotrophin regulation of synaptic transmission. Curr Opin Neurobiol 9:105-109.

Single S, Borst A (1998) Dendritic integration and its role in computing image velocity. Science 281:1848-1850.

Sjostrom PJ, Hausser M (2006) A cooperative switch determines the sign of synaptic plasticity in distal dendrites of neocortical pyramidal neurons. Neuron 51:227-238.

Sjostrom PJ, Nelson SB (2002) Spike timing, calcium signals and synaptic plasticity. Curr Opin Neurobiol 12:305-314.

Sjostrom PJ, Turrigiano GG, Nelson SB (2001) Rate, timing, and cooperativity jointly determine cortical synaptic plasticity. Neuron 32:1149-1164.

Spruston N, Schiller Y, Stuart G, Sakmann B (1995) Activity-dependent action potential invasion and calcium influx into hippocampal CA1 dendrites. Science 14:297-300.

Stuart G, Schiller J, Sakmann B (1997) Action potential initiation and propagation in rat neocortical pyramidal neurons. J Physiol (Lond) 505:617632. 